\title{
Emergence of Congenital Chagas Disease in Ireland and the UK
}

\author{
Roy Stone ${ }^{1}$, Patrick Gavin ${ }^{1}$, Debbie Nolder ${ }^{2}$, Padraig McGettrick ${ }^{3}$, Anna Keogh ${ }^{4}$, Noel Mc \\ Entagart $^{4}$, Richard Drew ${ }^{4}$, John Lambert ${ }^{3}$, and Wendy Ferguson ${ }^{1}$ \\ ${ }^{1}$ Children's Health Ireland at Temple Street \\ ${ }^{2}$ London School of Hygiene \& Tropical Medicine \\ ${ }^{3}$ Mater Misericordiae University Hospital \\ ${ }^{4}$ Rotunda Hospital
}

June 15, 2021

\section{Emergence of Congenital Chagas Disease in Ireland and the UK.}

Stone $\mathrm{RG}^{1}$, Gavin $\mathrm{P}^{1}$, Nolder $\mathrm{D}^{2}$, McGettrick $\mathrm{P}^{3}$, Keogh $\mathrm{A}^{4}$, Mc Entagart $\mathrm{N}^{4}$, Drew $\mathrm{R}^{5}$, Lambert $\mathrm{J}^{3}$, Ferguson $\mathrm{W}^{1}$

1. Paediatric Infectious Diseases, Rotunda Hospital and Children's Health Ireland at Temple Street, Dublin

2. Diagnostic Parasitology Laboratory, The London School of Hygiene and Tropical Medicine, London, UK

3. Infectious Diseases, The Mater Misericordiae University Hospital, Dublin

4. Histopathology, Rotunda Hospital, Dublin

5. Microbiology, The Rotunda Hospital, Dublin

\section{Corresponding author}

Name: Roy Gavin Stone

Email: roygavinstone@gmail.com

Address: Paediatric Infectious Diseases, Rotunda Hospital, Dublin

Telephone Number: 00353861574801

Short Title: Congenital Chagas Disease in Ireland \& UK

Key words : Chagas Disease, congenital infection, T.cruzi.

\section{Introduction}

Chagas disease (CD) or American Trypanosomiasis is a major public health problem in Latin America, where the disease burden is nearly 8 -times greater than that due to malaria. ${ }^{1}$ Almost 6 million people are infected worldwide. ${ }^{2} \mathrm{CD}$ is caused by infection with the protozoan parasite Trypanosoma cruzi (T. cruzi ). In endemic countries, T. cruzi is predominantly transmitted vectorially via the faeces of blood-sucking triatomine insects, also known as "kissing or assassin bugs". In non-endemic countries, infection may occur through blood transfusion, solid organ transplant or during pregnancy. Congenital Chagas disease (cCD) occurs in approximately $5 \%$ of chronically infected pregnant women. ${ }^{3}$ Chagas disease is emerging in Europe due to migration patterns. ${ }^{3,4}$ We report the first cases of cCD in Ireland and the United Kingdom (UK).

\section{Case Reports}


Case 1, a 3.56 kilogram $(\mathrm{kg})$ infant, was born at full-term to a Bolivian woman who had arrived in Ireland at 31 weeks gestation. She was diagnosed serologically with CD in Bolivia by routine antenatal screening at 23 weeks. Repeat maternal serology was tested at The Hospital for Tropical Diseases, London UK and was strongly positive.T. cruzi polymerase chain reaction (PCR) tested in the Diagnostic Parasitology Laboratory at The London School of Hygiene and Tropical Medicine, was also positive. As per World Health Organisation (WHO) recommendations, maternal trypanocidal treatment was delayed until after delivery. Newborn clinical examination was normal and microscopy of cord blood was negative for blood forms of T. cruzi . PCR of infant cord and venous blood (day 2) for T. cruzi were inconclusive (positive in 1 of 8 replicates and 2 of 12 replicates, respectively). Repeat PCR and microscopy of blood were negative at 4 weeks. Blood PCR for T. cruzi was strongly positive at 3 months of age and the infant was commenced on a 2-month course of oral Benznidazole 10 milligram $/ \mathrm{kg} /$ day. The infant experienced mild self-limiting gastrointestinal upset and macular rash at the onset of treatment. T. cruzi PCR was negative after one month of treatment and at treatment completion. Monitoring full blood count and liver function tests remained normal throughout treatment. T. cruzi PCR was also negative at 9 months of age.

The mother discontinued her Benznidazole treatment after 3 weeks due to concerns that it might interfere with breast-feeding and she disengaged from follow-up. Further maternal follow up is planned following cessation of breast feeding at one-year post-partum to recommence treatment and she has been advised to avoid pregnancy until treatment completion.

Case 2 was an infant born 4 months after case 1. Routine placental examination for intrauterine growth restriction (IUGR) in a $2.4 \mathrm{~kg}$ infant delivered by caesarean section at 39 weeks gestation, demonstrated numerous T. cruzi blood forms within the placental membranes and umbilical cord (Figure 1a). The infant, then aged 4 weeks, was recalled. Examination was normal save for symmetrical growth retardation. Presence of T. cruzi trypomastigotes in blood films (Figures $1 \mathrm{~b}$ and 1c) and positive blood PCR confirmed the diagnosis of cCD. The infant was commenced on a 2-month course of oral Benznidazole. Trypanocidal therapy was well tolerated. Iron supplementation was started at age 6 weeks for mild microcytic anaemia. PCR for T. cruzi and microscopy of venous blood were negative after 1 month of treatment and at treatment completion. The infant's mother was born in Bolivia and moved to Brazil at age 2 years. She migrated to Ireland 2 years prior to pregnancy. Twenty years earlier she was diagnosed serologically with CD in Brazil when family members had tested positive. She had attended annual cardiology review there but had not received trypanocidal treatment as her $\mathrm{CD}$ was asymptomatic. She chose to discontinue breast-feeding due to concerns of ongoing T. cruzitransmission. She was commenced on oral Benznidazole treatment and was referred for cardiology review.

Both infants are thriving, developing normally and will be monitored clinically and serologically up to age 1 year. Echocardiograms were normal and cardiology follow up is scheduled for both infants.

\section{Discussion}

Chagas disease is endemic in much of Central and South America, where the risk of congenital infection is recognised and routine antenatal screening is well established. ${ }^{2,}{ }^{5}$ Increasing migration from Latin America to Europe (and laterally to northern Europe) and the trend towards increased numbers of female migrants has increased the risk of congenital transmission from infected mothers in non-endemic countries; the highest incidence is reported in Spain. ${ }^{3}$ Murcia et. al. documented a transmission rate of $13.8 \%$ among pregnant seropositive Bolivian women in Spain. ${ }^{6}$

The UK is now home to around 250,000 people of Latin American origin. Approximately half live in London. ${ }^{7}$ While numbers of migrants from Latin America to Ireland are smaller in comparison, they are increasing; the Brazilian population in Ireland has more than trebled in size since $2006 .{ }^{8}$ Pooled prevalence of CD among Latin American migrants living in Europe is estimated to be $4 \%$ but the burden of disease is largely hidden. $^{3,4}$ It is estimated that $>95 \%$ of migrants to Europe infected with CD are undiagnosed. ${ }^{2,9}$ This report confirms the long held suspicion that changes in traditional migration patterns have increased the risk of $\mathrm{cCD}$ in Ireland and the UK. ${ }^{1}$ 
Both infants were born in 2020 to immunocompetent women from Bolivia, where seroprevalence rates are highest (40\% in some rural areas). ${ }^{2,3,6}$ Both women gave a history of asymptomatic chronic CD. Case 2 was complicated by IUGR, not surprising given the high parasitic burden observed in the placenta. ${ }^{10}$ Chagas disease increases the risk of preterm birth, low-birth weight and stillbirth - but otherwise there was little apparent cause for concern, and, as is commonly the case, both newborns were asymptomatic at birth. ${ }^{6,11}$ A minority of newborns with cCD have findings similar to those of congenital infection caused by classic TORCH (Toxoplasma gondii, Rubella, CMV and Herpes simplex) organisms. Recognition of the increased risk of cCD in Latin American migrants and the asymptomatic nature of maternal and neonatal infection has led Spain, France, Germany, Italy, and Switzerland to establish cost-effective targeted antenatal screening programs. ${ }^{4}$ In the continued absence of such initiatives in Ireland and the UK, diagnosis of cCD requires increased awareness to perform specific testing of mothers and infants in a timely manner with the assistance of specialised diagnostic centres. In the first case, notwithstanding a high index of suspicion based on positive antenatal serology and serial testing of the infant, congenital infection was not confirmed until 3 months of age. In the second case, despite a history of chronic maternal infection and IUGR, the diagnosis of cCD was unexpected; follow-up testing of the infant was triggered by findings on placental histology.

The consequences of a missed diagnosis of cCD can be severe. Left untreated, $40 \%$ of infants with cCD progress to chronic infection with symptomatic cardiac, neurological or gastrointestinal disease later in life and untreated female infants may perpetuate vertical transmission from one generation to the next. ${ }^{2}$ The gold standard for diagnosis of cCD is identification of parasites in cord or venous blood of the newborn or at any time in infancy, or positive $T$. cruzi specific serology in infants $>8$ months of age. ${ }^{5}$ Negative serology $>8$ months of age indicates the absence of congenital infection. PCR for T. cruziis available in specialised centres and has higher sensitivity than microscopy. ${ }^{2,11}$ As in Case 1, tests may be inconclusive in early congenital infection due to low initial parasite replication as parasitaemia peaks beyond 1 month of life and thus may need to be repeated serially in suspected cases. ${ }^{5,10}$

Congenital CD should be treated as soon as the diagnosis is confirmed. ${ }^{2}$ The teratogenic risks are unknown and adverse drug reactions are relatively common in adults, thus it is not possible to prevent congenital infection in established pregnancy. Whilst efficacy and safety of available anti-trypanosomal treatment (Benznidazole and Nifurtimox) in adult populations is far from ideal, treatment in early infancy is almost $100 \%$ effective and adverse drug reactions are rare. ${ }^{2,5,11}$ While the infants tolerated Benznidazole treatment well, both mothers had concerns related to breast-feeding. One mother discontinued her Benznidazole treatment because of concerns that it might reduce lactation and quality of breast milk, and the other discontinued breast-feeding because of concerns for ongoing $T$. cruzi transmission from mother to child via breast milk. Risk of transmission of T. cruzi in breast-milk is considered low and interruption of breast-feeding is generally not recommended but published studies are scarce. ${ }^{12}$ Pasteurisation or microwaving expressed breast milk is considered a safe alternative. Temporary interruption of breast-feeding is recommended if the mother has fissured or bleeding nipples.

The consequences of a missed or delayed diagnosis of cCD in infancy can be severe, but reliable tests for cCD are available and the condition is curable. In Ireland and the UK, structures exist for antenatal screening for other infectious diseases and capture of maternal demographics. Targeted testing of women who give a clear history of $\mathrm{CD}$ is a potential method for early diagnosis of cCD. In the absence of targeted screening programs, education of health care providers in Ireland and the UK regarding the potential risk of cCD in pregnant Latin American women would be a useful first step toward early detection and treatment of cCD.

\section{Acknowledgements}

We wish to thank: Professor Peter Chiodini, (Director of the Hospital for Tropical Diseases and London School of Hygiene and Tropical Medicine, UK) for prompt return of diagnostic test results and expert interpretation; Dr. Pedro Albajar Vinas and colleagues at the WHO Chagas Disease Division in Geneva Switzerland for their expert advice and rapid delivery of antiparasitic medication; the mothers of the infants for consenting to be included in this study. 


\section{Disclosure of interest}

Nothing to declare.

\section{Details of ethical approval}

Ethical approval was granted from the Rotunda Ethics Committee (Reference Number REC-2020-034) on the 20th November 2020.

\section{Funding}

None received.

\section{Contribution to authorship}

WF conceived the idea. RGS and WF performed data collection. DN contributed the infant images. AK, NMcE contributed placental images. RGS, PMcG, PG, RD, AK, NMcE, JL, DN and WF contributed to writing the manuscript and approved the final version.

\section{References}

1. Elkheir N, Carter J, García-Mingo A, Chiodini P. Chagas disease in non-endemic settings. BMJ. 2021:n901.

2. Pérez-Molina JA, Molina I. Chagas disease. The Lancet. 2018;391(10115):82-94.

3. Colombo V, Giacomelli A, Casazza G, Galimberti L, Bonazzetti C, Sabaini F, et al. Trypanosoma cruzi infection in Latin American pregnant women living outside endemic countries and frequency of congenital transmission: a systematic review and meta-analysis. Journal of Travel Medicine. 2021;28(1).

4. Requena-Méndez A, Aldasoro E, de Lazzari E, Sicuri E, Brown M, Moore DA, et al. Prevalence of Chagas disease in Latin-American migrants living in Europe: a systematic review and meta-analysis. PLoS Negl Trop Dis. 2015;9(2):e0003540.

5. Carlier Y, Altcheh J, Angheben A, Freilij H, Luquetti AO, Schijman AG, et al. Congenital Chagas disease: Updated recommendations for prevention, diagnosis, treatment, and follow-up of newborns and siblings, girls, women of childbearing age, and pregnant women. PLOS Neglected Tropical Diseases. 2019;13(10):e0007694.

6. Murcia L, Carrilero B, Munoz-Davila MJ, Thomas MC, López MC, Segovia M. Risk Factors and Primary Prevention of Congenital Chagas Disease in a Nonendemic Country. Clinical Infectious Diseases. 2013;56(4):496-502.

7. Bunge CMaD. Towards visibility: the Latin American community in London. London: Queen Mary University of London; 2016.

8. CSO. Census 2016 -Non-Irish Nationalities Living in Ireland CSO website: Central Statistics Office; 2016 [updated 2016; cited 2021 24th April]. Available from: https://www.cso.ie/en/releasesandpublications/ep/pcpnin/cpnin/brazilian/.

9. Requena-Méndez A, Moore DAJ, Subirà C, Muñoz J. Addressing the neglect: Chagas disease in London, UK. The Lancet Global Health. 2016;4(4):e231-e3.

10. Carlier Y, Truyens C. Congenital Chagas disease as an ecological model of interactions between Trypanosoma cruzi parasites, pregnant women, placenta and fetuses. Acta Tropica. 2015;151:103-15.

11. Klein MD, Tinajeros F, del Carmen Menduiña M, Málaga E, Condori BJ, Verástegui M, et al. Risk Factors for Maternal Chagas Disease and Vertical Transmission in a Bolivian Hospital. Clinical Infectious Diseases. 2020;ciaa1885

12. Norman FF, López-Vélez R. Chagas Disease and Breast-feeding. Emerging Infectious Diseases. $2013 ; 19(10)$.

\section{Hosted file}


Figure 1 Emergence of Congenital Chagas Disease in Ireland and the UK.docx

available at https://authorea.com/users/419928/articles/526313-emergence-of-congenital-chagasdisease-in-ireland-and-the-uk 